APUNTE

\title{
Productos Forestales No Madereros Presentes en los Bosques de Nirre (Nothofagus antarctica) de la Zona Sur Austral de Chile
}

\author{
Salinas, Jaime ${ }^{1 *}$ y Uribe, Alicia ${ }^{1}$ \\ Investigadores INFOR Sede Patagonia, Coyhaique. jsalinas@infor.cl auribe@infor.cl \\ *Autor para correspondencia \\ DOI: https://doi.org/10.52904/0718-4646.2021.474
}

Recibido: 01.04.21; Aceptado: 20.04.21

\section{RESUMEN}

Los bosques representan el entorno de gran parte del mundo rural y les proporcionan a sus pobladores una cantidad y variedad de Productos Forestales No Madereros (PFNM), que representan recursos alimenticios e insumos para diversos productos que esta población rural puede utilizar en forma ambiental y económicamente sostenible, para su propio beneficio o comercializar para un mejoramiento en sus condiciones de vida y sustento.

Los bosques de ñirre forman parte del territorio de Aysén y su distribución coincide con la frontera urbana y la ganadera, donde han sido sometidos por décadas a disturbios derivados de la acción antrópica. En estos bosques se encuentran diferentes PFNM, que son importantes para la alimentación y medicina de comunidades rurales, algunos de estos productos son el calafate, la rosa mosqueta, la morilla, el musgo, las hierbas medicinales, los follajes y semillas de árboles nativos, entre otros.

Este trabajo buscó investigar la diversidad actual de PFNM asociados a los bosques de ñirre de la zona sur austral de Chile, en base al conocimiento empírico y al uso de las comunidades. Para ello, se realizaron talleres participativos, con preguntas dirigidas a la expresión de las comunidades con respecto a los productos de cada sector, sus usos y utilidades para luego dar espacio a una priorización de los 3 PFNM de mayor importancia, tanto social como económica y cultural, en las comunidades consultadas. Se identificaron alrededor de 70 especies de uso no maderero presentes en los bosques de ñirre, con más de 100 diferentes usos para las personas, entre los que destacan usos medicinales, comestibles, tintóreos y ornamentales. Estas especies son colectadas por recolectores para consumo personal o comercialización. Las tres especies priorizadas por las comunidades fueron el hongo morchella, la frutilla silvestre y el calafate.

Palabras clave: Ñirre, comunidades rurales, usos medicinales

\section{SUMMARY}

Forests represent the environment of a large part of the rural world and provide their inhabitants with a quantity and variety of Non-Wood Forest Products (NWFP), which represent food resources and inputs for various products that this rural population can use in a way environmentally and economically sustainable, for their own benefit or to commercialize for an improvement in their living conditions and livelihoods.

The ñirre forests are part of the Aysén territory and their distribution coincides with the urban and livestock frontiers, where they have been subjected for decades to disturbances derived from anthropic action. In these forests live different NWFP are important for the nutrition and medicine of rural communities, some of these products are calafate, Rosehip, Morel, moss, medicinal herbs, foliage and seeds of native trees, among others.

This work was to research on the current diversity of NWFP associated with the ñirre forests of the southern zone of Chile, based on empirical knowledge and the use of the communities. To this end, participatory workshops were held, with questions aimed at the expression of the communities regarding the products of each sector, their uses and utilities to then give space to a prioritization of the 3 NWFP of greatest social, economic and cultural importance in the consulted communities. Around 70 species of non-timber use present in the ñirre forests were identified, with more than 100 different uses for people, among which are medicinal, edible, dyeing and ornamental uses. These species are collected by people for personal consumption or commercialization. The three species prioritized by the communities were the morchella fungus, wild Strawberry and calafate.

Key Words: Ñirre, rural communities, medicinal uses. 


\section{INTRODUCCIÓN}

Los Productos Forestales No Madereros (PFNM), son definidos como bienes de origen biológico, distintos de la madera, derivados del bosque, de otras áreas forestales y de los árboles fuera de los bosques (FAO, 2003).

En los últimos años las organizaciones gubernamentales, no gubernamentales y grupos científicos, han promovido la utilización de los PFNM, como un elemento determinante en la disminución de la pobreza y el manejo sostenible de los ecosistemas (Martínez, 2005).

En el mundo existe una gran cantidad y variedad de PFNM y cada uno aporta con diferentes usos y oficios y emprendimientos relacionadas con temas productivos, sociales y culturales, aportando a las economías locales, manteniendo habitadas zonas aisladas como ocurre en el caso de la región de Aysén.

En esta región existe una superficie de 4.398 .746 ha cubierta por bosques nativos, que representa el $41 \%$ del total nacional (CONAF, 2012), y de esta superficie los bosques de ñirre suman 131.593 ha (Salinas et al., 2017). El ñirre es una especie endémica de los bosques templados lluviosos o subantárticos de Chile y Argentina, y presenta el rango de distribución más amplio de los Nothofagus sudamericanos.

Se trata de una especie monoica que se presenta como árbol pequeño o arbusto achaparrado, aunque generalmente alcanza los $10 \mathrm{~m}$ de altura. Sin embargo, en condiciones óptimas de sitio puede alcanzar los $15 \mathrm{~m}$ y fustes de $60 \mathrm{~cm}$ de diámetro (Rodríguez et al., 1983; Donoso, 1974). Salinas et al. (2015) reportan alturas cercanas a los $23 \mathrm{~m}$ en suelos profundos de la comuna de Chile Chico, región de Aysén.

En esta región puede asociarse con lenga (Nothofagus pumilio (Poepp. \& Endl.) Krasser) y coigue (Nothofagus dombeyi (Mirb.) Oerst.), pero generalmente forma bosques puros y coetáneos denominados ñirrantales.

En los bosques de nirre se encuentran diferentes PFNM, algunos de estos productos son calafate, rosa mosqueta, morilla, musgo, hierbas medicinales, follajes y semillas de árboles nativos, entre otros.

A pesar de la diversidad de PFNM existentes, los distintos sectores sociales y productivos no logran reconocer más que solo algunos, como el hongo Morchella ssp. (Salinas et al., 2018) y frutos como mosqueta y calafate, esto debido a la falta de divulgación de los productos que cohabitan en las formaciones boscosas de ñirre y de sus usos, utilidad e importancia.

Además, el aprovechamiento sustentable de estos PFNM puede aportar a desarrollar más alternativas productivas para la Agricultura Familiar Campesina (AFC), ya que aumenta la diversificación.

Los PFNM encierran un gran potencial para el futuro desarrollo de las economías locales y regionales. En esta misma línea en Argentina hay ejemplos de uso de las hojas de ñirre, como compuestos antioxidantes y aceites esenciales con beneficios para la salud (propiedades nutracéuticas) y una potencialidad para el uso en la industria cosmética.

El uso de estos recursos necesita de definiciones técnicas que aseguren una colecta sustentable y la conservación de los bosques que los proveen. El reservorio de recursos que habitan en el bosque de ñirre son especies vegetales nativas, no investigadas en profundidad, que podrían ser utilizadas como ingredientes bioactivos para la creación, desarrollo y diversificación de nuevos alimentos o productos farmacéuticos, asociados al fortalecimiento de los territorios.

En el año 2017 se crea el Programa de Investigación silvícola y tecnológica en Productos Forestales No Madereros (PFNM) generados en ecosistemas boscosos y con este programa se pretende levantar, sistematizar y difundir el conocimiento de los PFNM que se asocian a ecosistemas boscosos de Chile, así como su uso, manejo y aprovechamiento por comunidades rurales.

El presente trabajo propone investigar la diversidad actual de PFNM asociados a los bosques de ñirre de la zona sur austral de Chile, en base al conocimiento empírico y al uso de las comunidades. 


\section{METODOLOGIA}

\section{Área de Estudio}

El presente trabajo se realizó en la comuna de Coyhaique, región de Aysén. Se seleccionó un territorio que comprende las comunidades de Villa Ortega, El Richard, Arroyo El Gato y Rodeo Los Palos (Figura 1). Estas zonas se caracterizan por presentar un clima de estepa fría de vertiente oriental de los Andes Patagónicos o Transandinos y está protegida por el cordón montañoso de la cordillera, la que permite una disminución notable de las precipitaciones en comparación con el sector de archipiélagos (clima oceánico) que se encuentran en la misma latitud.

Se tomaron registros de las variables climáticas imperantes, obtenidos desde la estación meteorológica permanente más cercana al territorio estudiado. Las variables registradas por los equipos meteorológicos fueron la temperatura media del aire (Figura 2), precipitaciones, temperatura del suelo y radiación solar (Figura 3). De acuerdo a la clasificación de Köppen, el clima de la región de Aysén corresponde a Trasandino con Degradación Esteparia y se caracteriza por precipitaciones medias de $1.200 \mathrm{~mm}$ (IRENCORFO, 1979) con un periodo corto de escasez de precipitación entre enero y febrero, y ocurrencia de vientos intensos que fluctúan entre 37 y $56 \mathrm{~km} / \mathrm{h}$, con ráfagas de $60-80 \mathrm{~km} / \mathrm{h}$ en época de primavera.

La temperatura muestra una marcada tendencia a medias mensuales inferiores a $10{ }^{\circ} \mathrm{C}$ gran parte del año. Durante los períodos de evaluación de ensayos, la temperatura media anual del año 2018 fue de 5,9 ${ }^{\circ} \mathrm{C}$. Las temperaturas mínimas se presentaron en los meses de junio y julio con promedios de $-0,5$ y $-0,4$ ${ }^{\circ} \mathrm{C}$, respectivamente. Si bien la temperatura podría generar una disminución de la temperatura del bosque por convección, la estructura del bosque y del suelo permite la mantención de estas un poco más altas y permitir el desarrollo y crecimiento normal para las especies adaptadas a estas condiciones del ambiente. En términos de precipitaciones, el mes de septiembre presentó los valores más altos de precipitación acumulada con valores de $106 \mathrm{~mm}$, mientras que diciembre fue el mes que presento menor precipitación acumulada de la temporada, con solo 13,1 mm/año. La precipitación acumulada anual fue de $707 \mathrm{~mm}$.

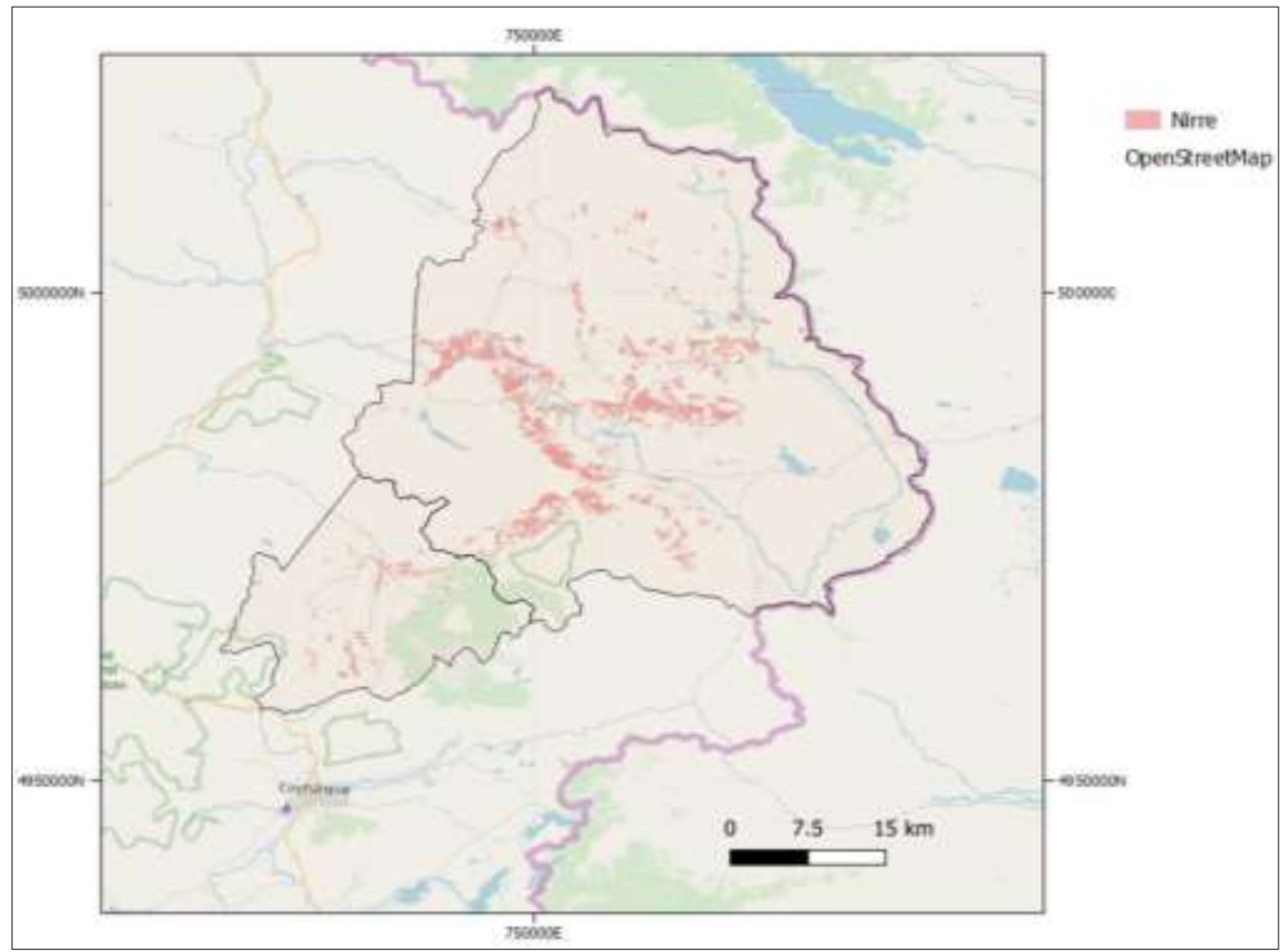

Figura 1. Distribución de los Bosques de Ñirre en Sitio de Estudio 


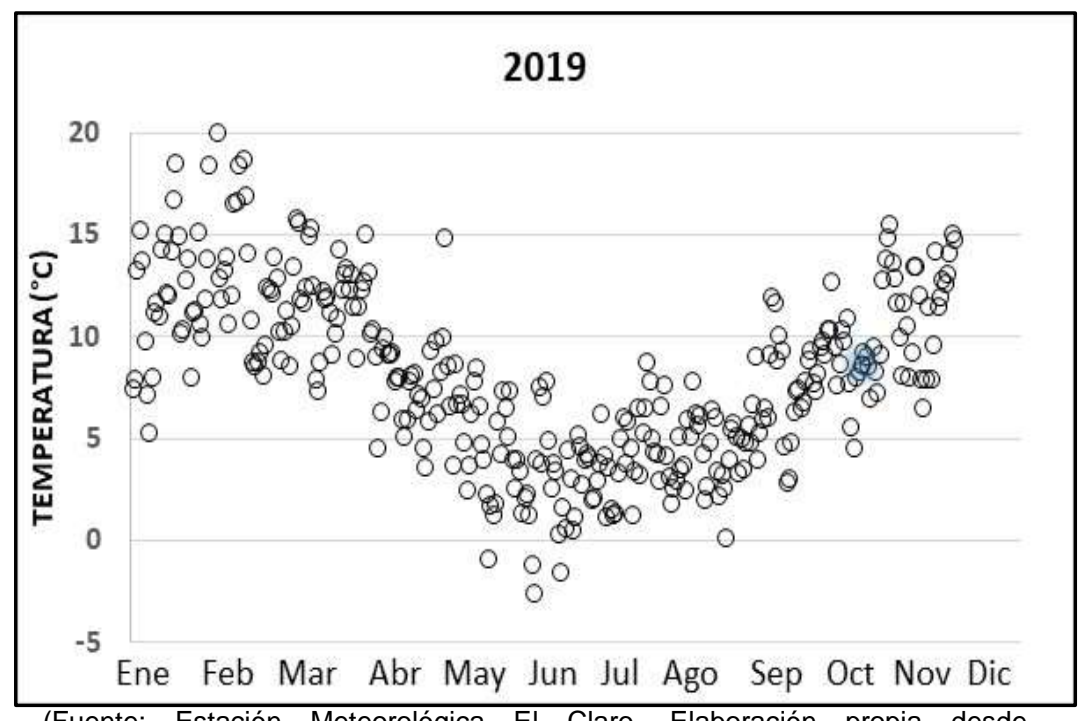

(Fuente: Estación Meteorológica El Claro. Elaboración propia desde http://agromet.inia.cl).

Figura 2. Temperaturas del Aire en Temporada 2019
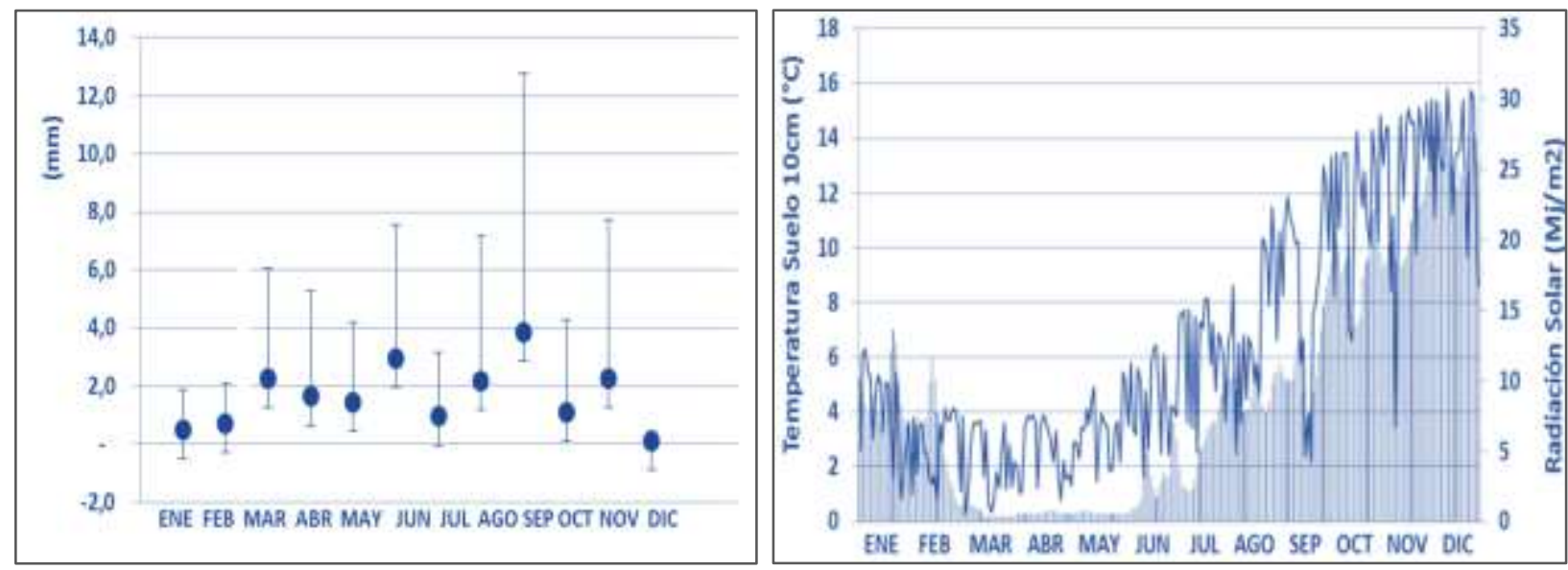

(Fuente: elaboración propia desde http://agromet.nia.cl)

Figura 3. Precipitación Anual, Temperatura del Suelo $(10 \mathrm{~cm})$ y Radiación Solar Durante la Temporada 2018, Estación Meteorológica El Claro

Finalmente, se presenta un gráfico sobre el Índice de Oscilación del Sur (SOI), que da una indicación del desarrollo y la intensidad de los eventos de El Niño o La Niña en el Océano Pacífico. El SOI se calcula utilizando las diferencias de presión entre Tahití y Darwin.

Los valores negativos sostenidos del SOI inferiores a -7 a menudo indican episodios de El Niño. Estos valores negativos generalmente van acompañados de un calentamiento sostenido del Océano Pacífico, una disminución de la fuerza de los vientos alisios del Pacífico y una reducción de las lluvias de invierno y primavera.

Los valores positivos sostenidos de SOI superiores a +7 son típicos de un episodio de La Niña y están asociados con vientos alisios más fuertes del Pacífico y temperaturas más cálidas del mar. 


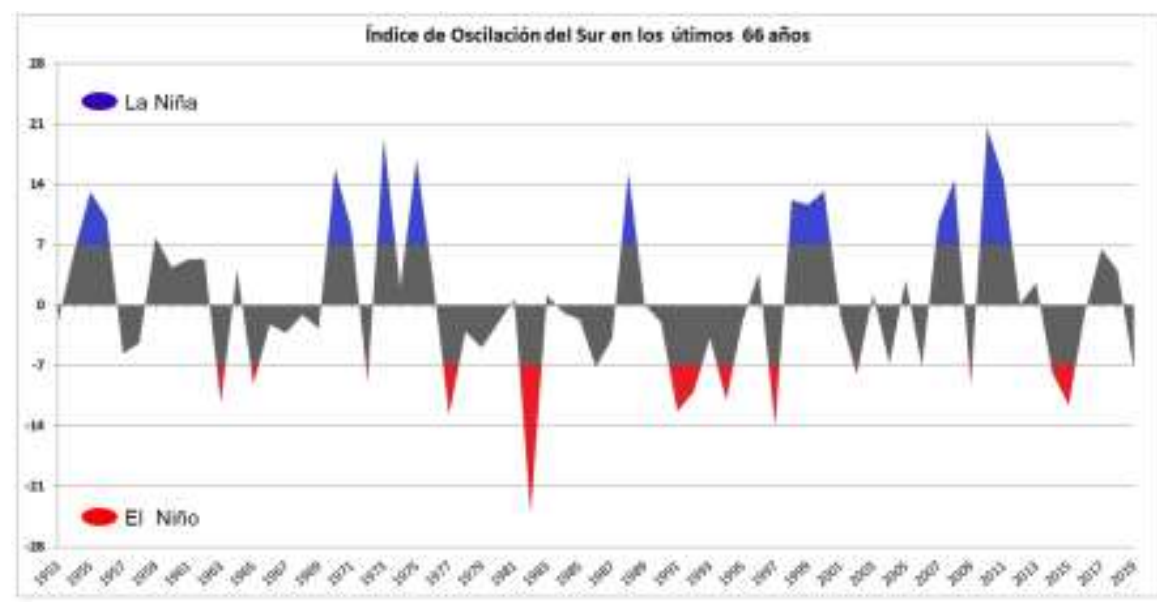

(Fuente: Oficina De Meteorología De Australia http://www.bom.gov.au/climate/current/soihtm1.shtml).

Figura 4. Índice de Oscilación del Sur (SOI) en los Últimos 66 Años

\section{Levantamiento de Información}

Se revisó y recopiló información disponible de los PFNM identificados, de acuerdo a un formato estándar, la información básica sobre los productos y recursos, así como valor económico. Lo anterior, generando herramientas de divulgación y preservación del bosque nativo con la comunidad en las poblaciones cercanas a los puntos de colecta y los pequeños empresarios interesados en el rubro.

El objetivo es proporcionar una categorización de los PFNM identificados en el ñirrantal, con recopilación de información en fuentes primarias, secundarias y terciarias. Se recopiló la información de las especies en biblioteca, páginas WEB y comunicaciones personales.

Luego se analizó la información en tres aspectos, localización, discriminación y selección entre todas las fuentes recopiladas, y la información útil respecto de lo que se desea incluir en la cartilla divulgativa.

Se caracterizó el sitio donde se colectó la información a través de visitas y muestreos de las características. El sitio de estudio abarcó un área vegetacional de la ecorregión andino boreal, expresándose en tres tipos fisionómicos.

Al Oeste, se presenta el bosque caducifolio, al Este se define una vegetación altamente homogénea de estepas con gramíneas en mechón o coirones (Festuca spp. y Stipa spp.), y en la posición intermedia se ubica el bosque de y matorrales, con una fuerte penetración de elementos florísticos de los bosques andino patagónicos.

\section{RESULTADOS}

\section{Caracterización de la Especie Ñirre}

El ñirre es un árbol nativo de Chile y Argentina, es una especie monoica que se presenta como arbolito pequeño o arbusto achaparrado, aunque generalmente alcanza los $10 \mathrm{~m}$ de altura (Figura 5). Sin embargo, en condiciones óptimas de sitio puede alcanzar los $15 \mathrm{~m}$ y fustes de $60 \mathrm{~cm}$ de diámetro (Rodríguez et al. 1983; Donoso, 1974).

Diferentes morfotipos han sido identificados para la especie en Chile y diferentes ecotipos han sido sugeridos dentro del parque Nacional Nahuel Huapi en el noreste de la Patagonia Argentina en donde ñirre crece en cuatro tipos de hábitats, presentando distintas características arquitectónicas para su crecimiento individual en contraste con cada entorno físico (Steinke et al., 2008). 
Taxonómicamente presenta la siguiente clasificación:

$\begin{array}{ll}\text { Reino } & : \text { Plantae } \\ \text { División } & : \text { Magnoliophyta } \\ \text { Clase } & : \text { Magnoliopsida } \\ \text { Sub-clase } & : \text { Hammamelidae } \\ \text { Orden } & : \text { Fagales } \\ \text { Familia } & : \text { Fagaceae } \\ \text { Género } & : \text { Nothofagus } \\ \text { Especie } & : \text { Nothofagus antarctica } \\ \text { Nombre común } & : \text { Ñire, ñirre o haya antarctica }\end{array}$

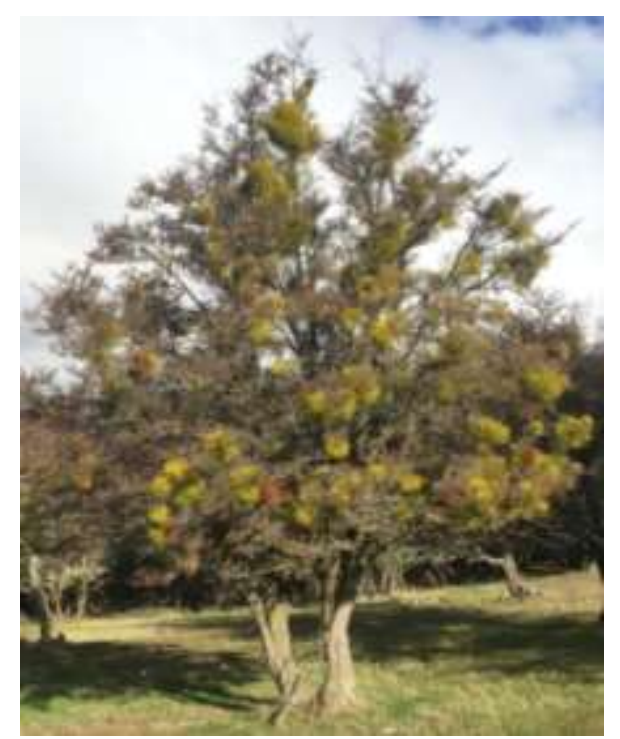

Figura 5. Individuo de Ñirre con Presencia de Misodendrum sp.

\section{Descripción Fenotípica}

Esta especie exhibe polimorfismo intraespecífico, el cual se ha asociado a una adaptación de la especie a los diferentes biotopos, modificando su cuerpo vegetativo y adoptando distintas formas de vida (Donoso, 2006). Según Romero (1986) estos ecotipos podrían ser resultado de presiones de selección relativamente recientes tendientes a la especiación. Es considerada la especie de Nothofagus de Sudamérica con mayor variación morfológica y de mayor tolerancia ecológica (Ramírez et al., 1985). En Chile se distinguen tres morfotipos distintos del ñirre, el primero arbóreo en condiciones óptimas, uno arbustivo achaparrado (Krummholz) en terrenos pedregosos o clima riguroso y un último como arbustivo caméfito de turbera (Ramírez et al., 1985).

De tal forma que en hábitats más favorables, protegidos del viento, con mayor humedad y suelos bien drenados y fértiles, alcanza porte arbóreo y puede dar lugar a formaciones puras (Navarro Cerrillo et al., 2008), sin embargo, también muestra un buen desempeño en suelos anegados, como señala Donoso (1987), en ñadis del llano central el ñirre puede crecer en condiciones óptimas. En Patagonia, el ñirre domina las tierras bajas, zonas de valle y aquellos terrenos que han sido perturbados por incendios 0 ganado (Armesto et al., 1992).

Ramírez et al. (1985) también señalan que la capacidad, o plasticidad, que tiene esta especie de colonizar sitios pobres es explicada, en gran parte, por la poca capacidad de competencia del ñirre, por lo que, en mejores sitios, en donde se podría desarrollar en forma óptima, es usualmente desplazada por otras especies. Es por esto que, si bien se desarrolla en sitios extremos (pedregosos, suelos pobres, etc.), presenta una pérdida de vitalidad y capacidad de reproducción sexual dando lugar a la reproducción vegetativa como forma más común de regeneración (Mc Queen, 1977). Observaciones realizadas en terreno sugieren que en sitios donde ñirre coexiste simpátricamente con lenga se habrían 
detectado individuos con morfología foliar intermedia entre ambas especies, estos serían posibles híbridos que se formarían en áreas donde se sobreponen los rangos de distribución de ambas especies (Donoso, 2006).

La corteza es de color gris, rugoso, áspero y muy agrietado longitudinalmente en forma irregular. Es una especie multiramificada, con hojas deciduas al igual que roble (Nothofagus obliqua (Mirb.) Oerst.) y lenga (Mc Queen, 1977). Sus flores son pequeñas y unisexuales. (Dimitri y Milano 1950 cit. por Burns et al., 2010; Domínguez Díaz, 2012). Presenta hojas pequeñas (0,6 a 3,5 cm de largo), aovado-redondeadas a oblongas, con base acorazonada; bordes finamente dentados, lobulados y ondulados. Presenta flores femeninas y masculinas y frutos formados por tres pequeñas nueces, de las cuales dos son triangulares, las que rodean a una plana que se sitúa en el centro (Hoffmann, 1997).

Entre los meses de abril y mayo el ñirre comienza a manifestar cambios de coloración de las hojas producto del receso invernal. Dependiendo de diversos factores (altitud, régimen hídrico, heladas, viento entre otros) la coloración comienza antes en algunas zonas geográficas. Por lo general en sitios de mayor altitud y menor temperatura promedio, el comienzo de la coloración y posterior caída de hojas se adelanta. Se distinguen cuatro colores en la etapa de coloración en ñirre; el color correspondiente a todo el periodo vegetativo es verde, cambiando a color rojizo, anaranjado y amarillo.

\section{Recopilación de Información de Terreno}

Se recogió información de fuentes primarias y secundarias (Figura 6). En base a fuentes primarias se visitaron los territorios vinculados con los bosques de ñirre y se aplicaron entrevistas personales y talleres con la comunidad.
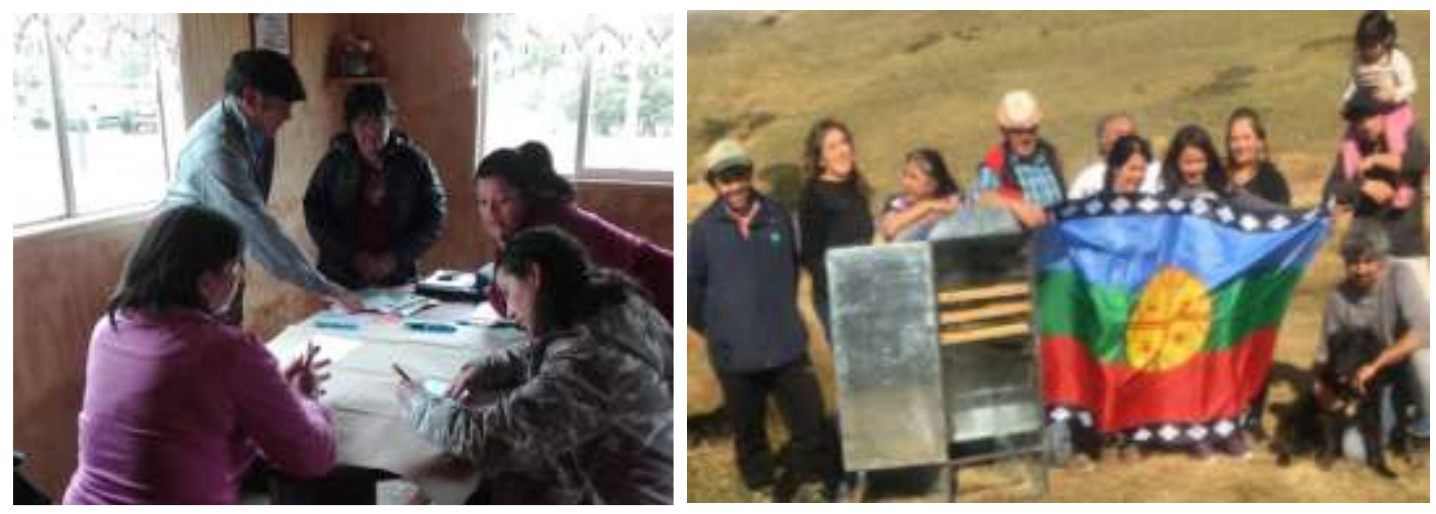

Figura 6. Rescate de Información en Comunidad de Villa Ortega y Comunidad Indígena Saihueque

Las acciones para conocer los PFNM que más valoran las comunidades tuvieron un enfoque participativo, el cual permitió validar los resultados con las comunidades locales, realizar adecuaciones e incorporar sus observaciones.

Al consultar a las comunidades de recolectores sobre los PFNM que son recolectados, un número apreciable de ellos mencionó las hierbas medicinales, su recolección, usos y utilización en medicina casera. Sin duda esta información entregada es relevante y parte de la sabiduría acumulada de las recolectoras.

\section{Listado de PFNM en Bosques de Ñirre}

Se logró cuantificar 74 especies de uso no maderero en los bosques de ñirre (Cuadro 1), muchas de ellas con más de un uso no maderero. De estas, 40 especies son de uso medicinal, 36 comestibles, 16 ornamental y 8 tintóreas. Del total un $63 \%$ son especies nativas, un $35 \%$ exóticas y un $2 \%$ cosmopolitas. Las principales familias fueron Asteraceae y Rosaceae con 17 y 9 especies, respectivamente. 
Cuadro 1. Catastro de Especies de los Bosques de Ñirre y Uso No Maderero

\begin{tabular}{|c|c|c|c|c|c|}
\hline Uso No Maderero & Nombre Científico & Nombre Común & Familia & Origen & Estructura Utilizada \\
\hline \multirow{40}{*}{ Comestible } & Nothofagus antarctica (G. Forst.) Oerst. & Nirre & Nothofagaceae & Nativa & Flores, hojas \\
\hline & Artemisia absinthium L. & Ajenjo & Asteraceae & Exótica & Flores, hojas \\
\hline & Trifolium pratense L. & Trébol rosado & Fabaceae & Exótica & Hojas, semillas \\
\hline & Erodium cicutarium (L.) L'Hér. ex Aiton & Alfilerillo & Geraniaceae & Exótica & Hojas \\
\hline & Alstroemeria aurea Graham & Amancay & Alstromeriaceae & Nativa & Flores, raíz \\
\hline & Osmorhiza chilensis Hook \& Arn. & Perejil del monte & Apiaceae & Nativa & Hojas, raíz \\
\hline & Berberis microphylla G. Forst. & Calafate & Berberidaceae & Nativa & Frutos \\
\hline & Berberis darwinii Hook & Michay & Berberidaceae & Nativa & Frutos \\
\hline & Chusquea culeou Desv. & Coligüe & Poaceae & Nativa & Brotes, semillas \\
\hline & Stellaria media (L.) Cirillo var. media & Hierba pajarera & Cariofilaceae & Exótica & Flores, hojas, tallos \\
\hline & Carduus thoermeri Weinm & Cardo & Asteraceae & Exótica & Tallos \\
\hline & Cirsium vulgare (Savi) Ten. & Cardo negro & Asteraceae & Exótica & Flores, hojas, tallos, raíz \\
\hline & Gaultheria mucronata (L. f.) Hook. \& Arn. & Chaura & Ericaceae & Nativa & Frutos \\
\hline & Gaultheria pumila (L. f.) D.J. & Chaura & Ericaceae & Nativa & Frutos \\
\hline & Sanicula graveolens Poepp. ex DC. & Cilantro silvestre & Apiaceae & Nativa & Frutos, hojas \\
\hline & Taraxacum officinale Weber ex F.H. Wigg. & Diente de león & Asteraceae & Exótica & Flores, hojas, tallos, raíz \\
\hline & Fragaria chiloensis (L) Duch & Frutilla silvestre & Rosaceae & Nativa & Frutos, hojas \\
\hline & Hypochaeris radicata L. & Hierba de chancho & Asteraceae & Exótica & Frutos, hojas \\
\hline & Prunella vulgaris L. & Hierba mora & Lamiaceae & Exótica & Hojas, tallos \\
\hline & Rumex crispus L. & Lengua de vaca & Poligonaceae & Exótica & Hojas, raíz \\
\hline & Plantago major L. & Llantén & Plantaginaceae & Exótica & Hojas \\
\hline & Plantago lanceolata L. & Siete venas & Plantaginaceae & Exótica & Hojas \\
\hline & Cyttaria darwinni Berkeley & LLao llao & Citariaceae & Nativa & Cuerpo fructífero \\
\hline & Cyttaria harioti Fischer & LLao llao & Citariaceae & Nativa & Cuerpo fructífero \\
\hline & Arjona tuberosa Cav. & Macachín & Schoepfiaceae & Nativa & Tubérculos \\
\hline & Madia sativa Mol. & Melosa & Asteraceae & Nativa & Semillas \\
\hline & Achillea millefolium L. & Milenrama & Asteraceae & Exótica & Flores, hojas \\
\hline & Ribes magellanicum Poir. & Parrilla & Grosulariaceae & Nativa & Frutos, hojas, tallos, raíz \\
\hline & Ribes cucullatum Hook. \& Arn. & Parrillita & Grosulariaceae & Nativa & Corteza, frutos, hojas \\
\hline & Galium aparine L. & Lengua de gato & Rubiaceae & Exótica & Brotes, semillas \\
\hline & Rosa canina L. & Rosa mosqueta & Rosaceae & Exótica & Flores, frutos \\
\hline & Rosa rubiginosa L. & Rosa mosqueta & Rosaceae & Exótica & Flores, frutos \\
\hline & Verbascum thapsus L. & Paño & Escrofulariaceae & Exótica & Flores, frutos \\
\hline & Trifolium repens L. & Trébol blanco & Fabaceae & Exótica & Flor, hojas, raíz, semillas \\
\hline & Rumex acetosella L. & Vinagrillo & Poligonaceae & Exótica & Hojas, raíz, semillas \\
\hline & Morchella sp. & Morchela & Morchellaceae & Cosmopolita & Cuerpo fructífero \\
\hline & Lepus europaeus & Liebre & Leporidae & Exótica & Todo cuerpo \\
\hline & Sus scrofa L. & Jabalí & Suidae & Exótica & Todo cuerpo \\
\hline & Phasianus colchicus L. & Faisán & Phasianidae & Exótica & Todo cuerpo \\
\hline & Cervus elaphus L. & Ciervo rojo & Cervidae & Exótica & Todo cuerpo \\
\hline
\end{tabular}




\begin{tabular}{|c|c|c|c|c|c|}
\hline \multirow{40}{*}{ Medicinal } & Artemisia absinthium L. & Ajenjo & Asteraceae & Exótica & Flores, hojas \\
\hline & Colletia hystrix Clos & Llaqui & Ramnaceae & Nativa & Ramas \\
\hline & Erodium cicutarium (L.) L'Hér. ex Aiton & Alfilerillo & Geraniaceae & Exótica & Flores, hojas \\
\hline & Alstroemeria aurea Graham & Amancay & Alstromeriaceae & Nativa & Flores, raíz \\
\hline & Protousnea sp. & Barba de viejo & Usneaceae & Nativa & Planta \\
\hline & Usnea sp. & Barba de viejo & Usneaceae & Nativa & Planta \\
\hline & Acaena splendens Gillies ex Hook \& Arn. & Cepa caballo & Rosaceae & Nativa & Planta \\
\hline & Discaria chacaye (G. Don) Tortosa & Chacay & Ramnaceae & Nativa & Corteza \\
\hline & Phacelia secunda J.F. Gmel. & Champa de oro & Borraginaceae & Nativa & Hojas \\
\hline & Escallonia virgata (Ruiz \& Pav.) Pers. & Chapel & Escaloniaceae & Nativa & Planta \\
\hline & Sanicula graveolens Poepp. ex DC. & Cilantro silvestre & Apiaceae & Nativa & Frutos \\
\hline & Taraxacum officinale Weber ex F.H. Wigg. & Diente de león & Asteraceae & Exótica & flores, hojas, raíz, tallos \\
\hline & Fragaria chiloensis (L) Duch & Frutilla silvestre & Rosaceae & Nativa & Hojas, raíz \\
\hline & Geranium sessiliflorum Cav. & Geranio & Geraniaceae & Nativa & Planta \\
\hline & Rumex crispus L. & Lengua de vaca & Poligonaceae & Exótica & Hojas, raíz \\
\hline & Equisetum bogotense Kunth & Limpia plata & Equisetaceae & Nativa & Planta \\
\hline & Plantago major L. & Llantén & Plantaginaceae & Exótica & Hojas, semillas \\
\hline & Plantago lanceolata L. & Siete venas & Plantaginaceae & Exótica & Hojas, semillas \\
\hline & Cyttaria harioti Fischer & LLao llao & Citariaceae & Nativa & Cuerpo fructífero \\
\hline & Matricaria recutita $\mathrm{L}$. & Manzanilla & Asteraceae & Exótica & Flores \\
\hline & Madia sativa Mol. & Melosa & Asteraceae & Nativa & Planta \\
\hline & Achillea millefolium $\mathrm{L}$. & Milenrama & Asteraceae & Exótica & Flores, hojas \\
\hline & Mutisia decurrens Cav. & Clavel del campo & Asteraceae & Nativa & Raíz \\
\hline & Mutisia spinosa Ruiz \& Pav. & Reina mora & Asteraceae & Nativa & Raíz \\
\hline & Mulinum spinosum (Cav.) Pers. & Neneo & Apiaceae & Nativa & Raíz \\
\hline & Fabiana imbricata Ruiz \& Pav. & Palo de piche & Solanaceae & Nativa & Corteza, hojas, raíz \\
\hline & Buddleja globosa Hope & Matico & Budlejaceae & Nativa & Hojas \\
\hline & Ribes magellanicum Poir. & Parrilla & Grosulariaceae & Nativa & Hojas, raíz, tallos \\
\hline & Ribes cucullatum Hook. \& Arn. & Parrillita & Grosulariaceae & Nativa & Corteza, hojas \\
\hline & Galium aparine L. & Lengua de gato & Rubiaceae & Exótica & Planta \\
\hline & Euphorbia collina Phil. var. collina & Pichoga & Euforbiaceae & Nativa & Hojas, tallos \\
\hline & Acaena pinnatifida Ruiz \& Pav. & Pimpinela & Rosaceae & Nativa & Hojas \\
\hline & Quinchimalium chilense Mol. & Quinchimalí & Schoepfiaceae & Nativa & Planta \\
\hline & Rosa canina L. & Rosa mosqueta & Rosaceae & Exótica & Flores, frutos \\
\hline & Rosa rubiginosa L. & Rosa mosqueta & Rosaceae & Exótica & Flores, frutos \\
\hline & Armeria maritima (Mill.) Willd. & Siempreviva & Plumbaginaceae & Nativa & Planta \\
\hline & Calceolaria polyrrhiza Cav & Capachito & Calceolariaceae & Nativa & Hojas \\
\hline & Calceolaria biflora Lam. & Capachito & Calceolariáceas & Nativa & Hojas \\
\hline & Trifolium repens $\mathrm{L}$. & Trébol blanco & Fabaceae & Exótica & Planta \\
\hline & Rumex acetosella L. & Vinagrillo & Poligonaceae & Exótica & Hojas, tallos, raíz \\
\hline \multirow{16}{*}{ Ornamental } & Alstroemeria aurea Graham & Amancay & Alstromeriaceae & Nativa & Flores, raíz \\
\hline & Acaena ovalifolia Ruiz \& Pav. & Cadillo & Rosaceae & Nativa & Planta \\
\hline & Senecio filaginoides DC. & Mata mora & Asteraceae & Nativa & Planta \\
\hline & Cystopteris fragilis (L.) Bernh. & Helecho & Dryopteridaceae & Nativa & Planta \\
\hline & Rumohra adiantiformis (G. Forst.) Ching & Helecho principal & Dryopteridaceae & Nativa & Planta \\
\hline & Mutisia decurrens Cav. & Clavel del campo & Asteraceae & Nativa & Planta \\
\hline & Mutisia spinosa Ruiz \& Pav. & Reina mora & Asteraceae & Nativa & Planta \\
\hline & Myosotis scorpioides L. & Nomeolvides & Boraginaceae & Exótica & Planta \\
\hline & Chloraea magellanica Hook. F. & Orquídea & Orquidaceae & Nativa & Planta \\
\hline & Armeria maritima (Mill.) Willd. & Siempreviva & Plumbaginaceae & Nativa & Planta \\
\hline & Vicia nigricans Hook. \& Arn & Vicia & Fabaceae & Nativa & Planta \\
\hline & Viola maculata Cav. var. maculata & Violeta de campo & Violaceae & Nativa & Planta \\
\hline & Calceolaria filicaulis Clos ssp. filicaulis & Capachito & Escrofulariaceae & Nativa & Planta \\
\hline & Berberis microphylla G. Forst. & Calafate & Berberidaceae & Nativa & Frutos, raíz \\
\hline & Blechnum penna-marina (Poir.) Kuhn & Punque & Blechnaceae & Cosmopolita & Planta \\
\hline & Anemone multifida Poir. & Anémona & Ranunculaceae & Nativa & Flores \\
\hline \multirow{8}{*}{ Tintóreas } & Nothofagus antarctica (G. Forst.) Oerst. & Ñirre & Nothofagaceae & Nativa & Corteza, hojas \\
\hline & Berberis darwinii Hook & Michay & Berberidaceae & Nativa & Frutos, raíz \\
\hline & Galium hypocarpium (L.) Endl. ex Griseb & Coral & Rubiaceae & Nativa & Raíz \\
\hline & Schinus patagonicus (Phil.) & Laura & Anacardiaceae & Nativa & Corteza \\
\hline & Rumex crispus L. & Lengua de vaca & Poligonaceae & Exótica & Hojas \\
\hline & Fabiana imbricata Ruiz \& Pav. & Palo de piche & Solanaceae & Nativa & Hojas, tallos \\
\hline & Buddleja globosa Hope & Matico & Budlejaceae & Nativa & Hojas \\
\hline & Rumex acetosella L. & Vinagrillo & Poligonaceae & Exótica & Hojas, tallo, raíz \\
\hline
\end{tabular}

(Información obtenida de muestreo en terreno, complementada desde Hansen et al., 2013) 


\section{Priorización de PFNM del Bosque de Ñirre Realizada por las Comunidades}

Para el ejercicio de priorización de los PFNM que tienen una mayor importancia y cuidado en el medio, se definió un listado de todos los más nombrados en cada reunión. En reunión siguiente las recolectoras se dividieron en dos grupos y con un paleógrafo realizaron un mapeo donde revisaron ubicación de los PFNM en sus respectivos sectores, finalmente se llegó a un consenso general para definir los 3 PFNM de mayor importancia en los bosques de ñirre de su territorio; morchella, frutilla silvestre y calafate. El enfoque participativo permitió así validar los resultados con las comunidades locales.

\begin{tabular}{lll}
\hline Nombre Común & Nombre Científico & llustraciones \\
\hline Morchella, morrilla & Morchella spp. & \\
& &
\end{tabular}
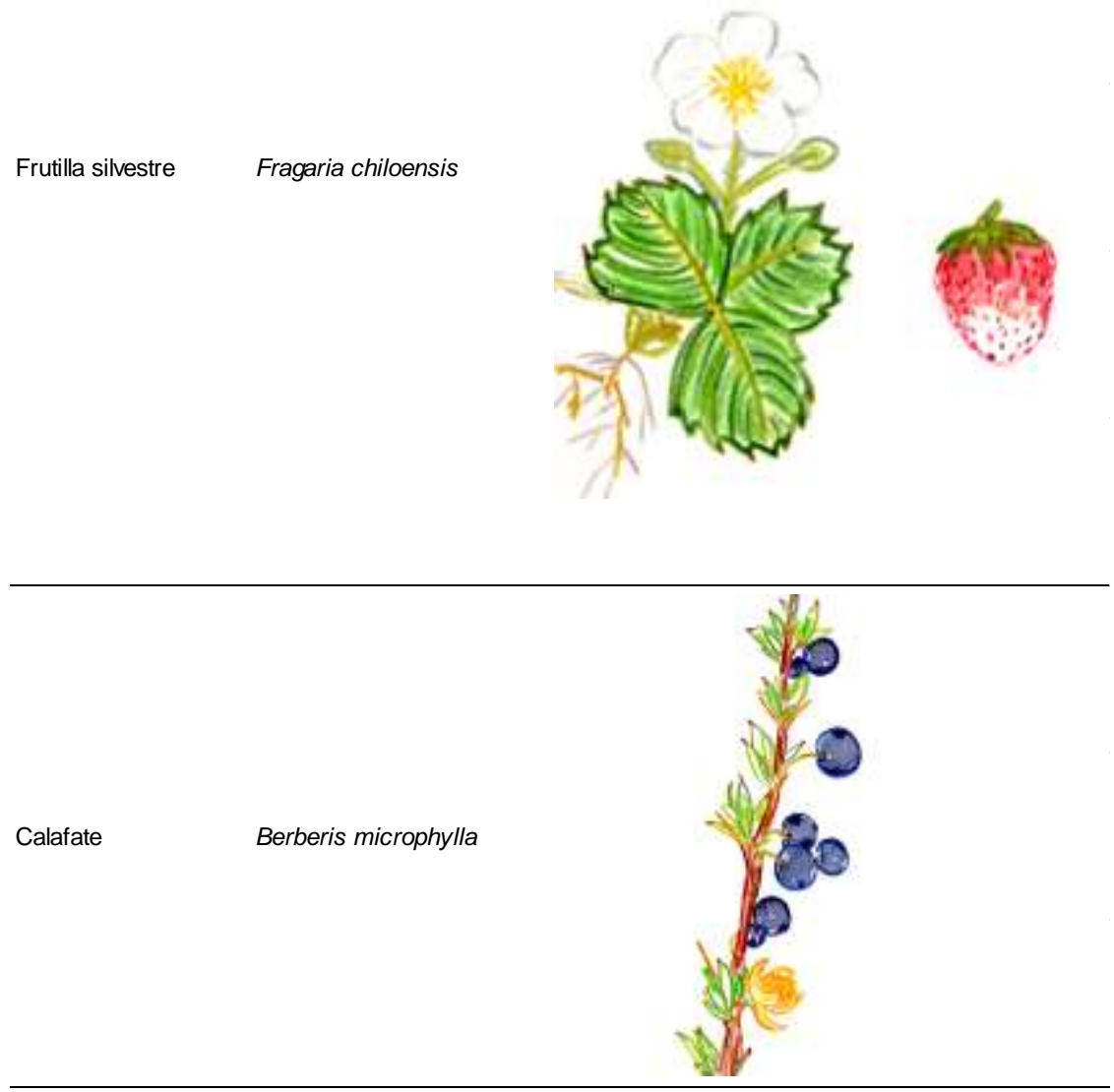

(Ilustraciones Catalina Mekis) 
A continuación, se presenta un resumen de información entregada por las recolectoras entrevistadas, donde exponen el uso de algunas especies de los bosques de ñirre.

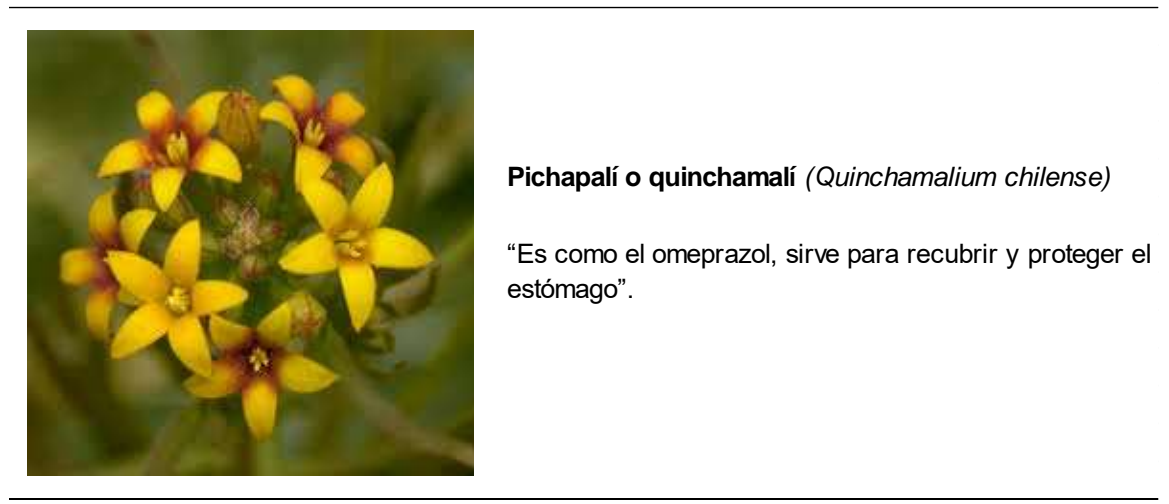

Cadillo (Acaena splendens)

"La ocupan para los riñones. También se utiliza para el pulmón, cuando los niños tienen neumonía y están muy congestionados".
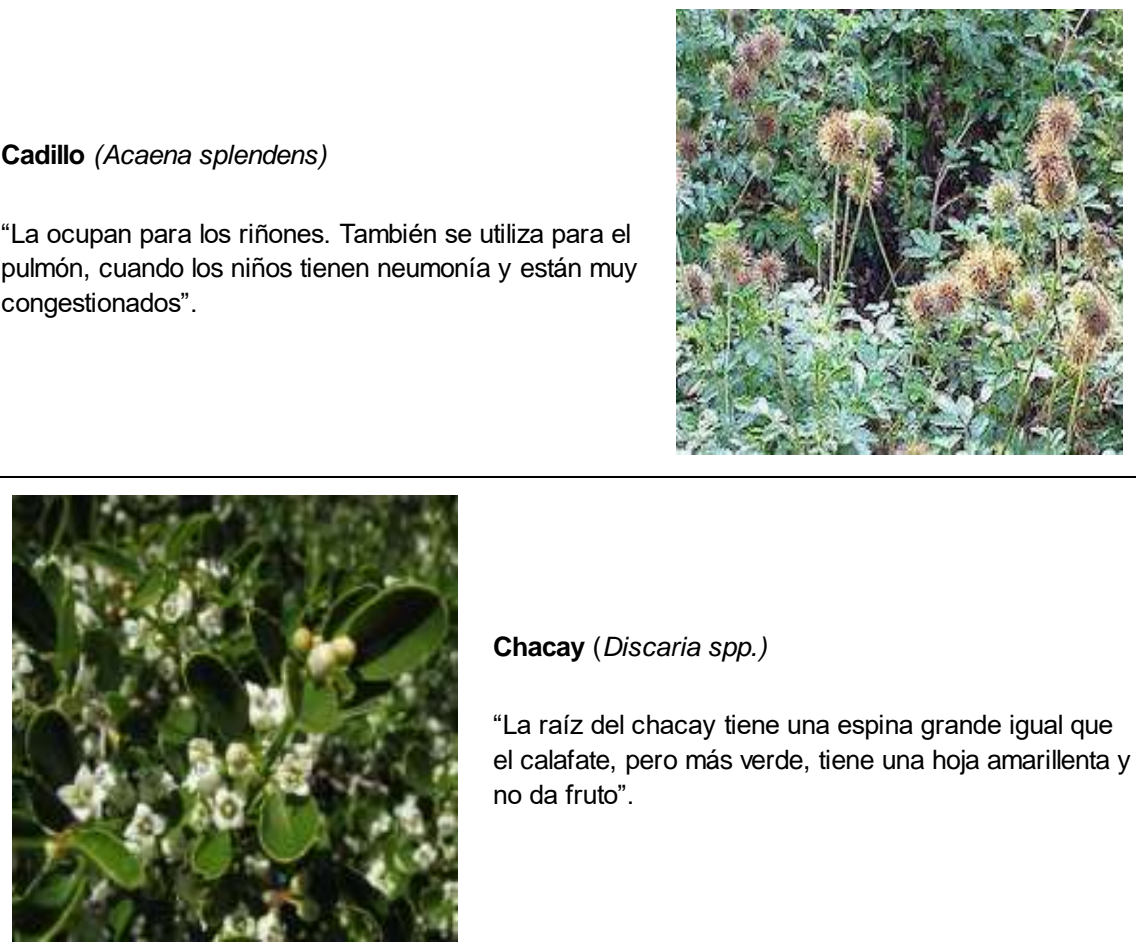

Chacay (Discaria spp.)

"La raíz del chacay tiene una espina grande igual que el calafate, pero más verde, tiene una hoja amarillenta y no da fruto".

Romasa (Rumex conglomeratus)

"Se toma en ayunas, con azúcar quemada, para el hígado y la vesícula".

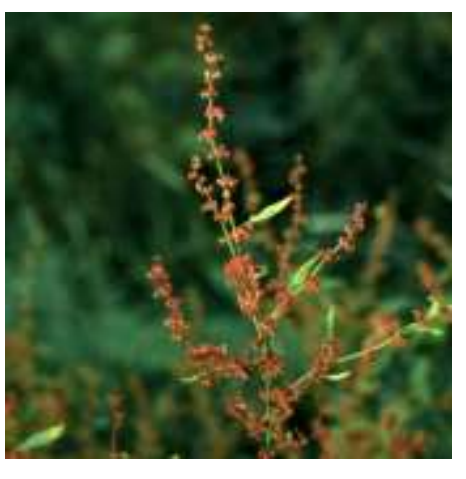




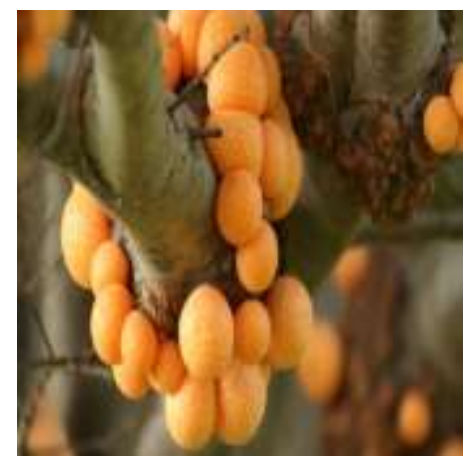

Digüeñe (Cyttaria sp.)

"Tiempo atrás era muy común encontrarlo en los árboles de lenga y ñirre, se comía como ensalada o solo con sal, ahora no es muy consumido ya que no crece mucho".

Sanguinaria (Sanguinaria canadensises)

"Se utilizada para la sangre".
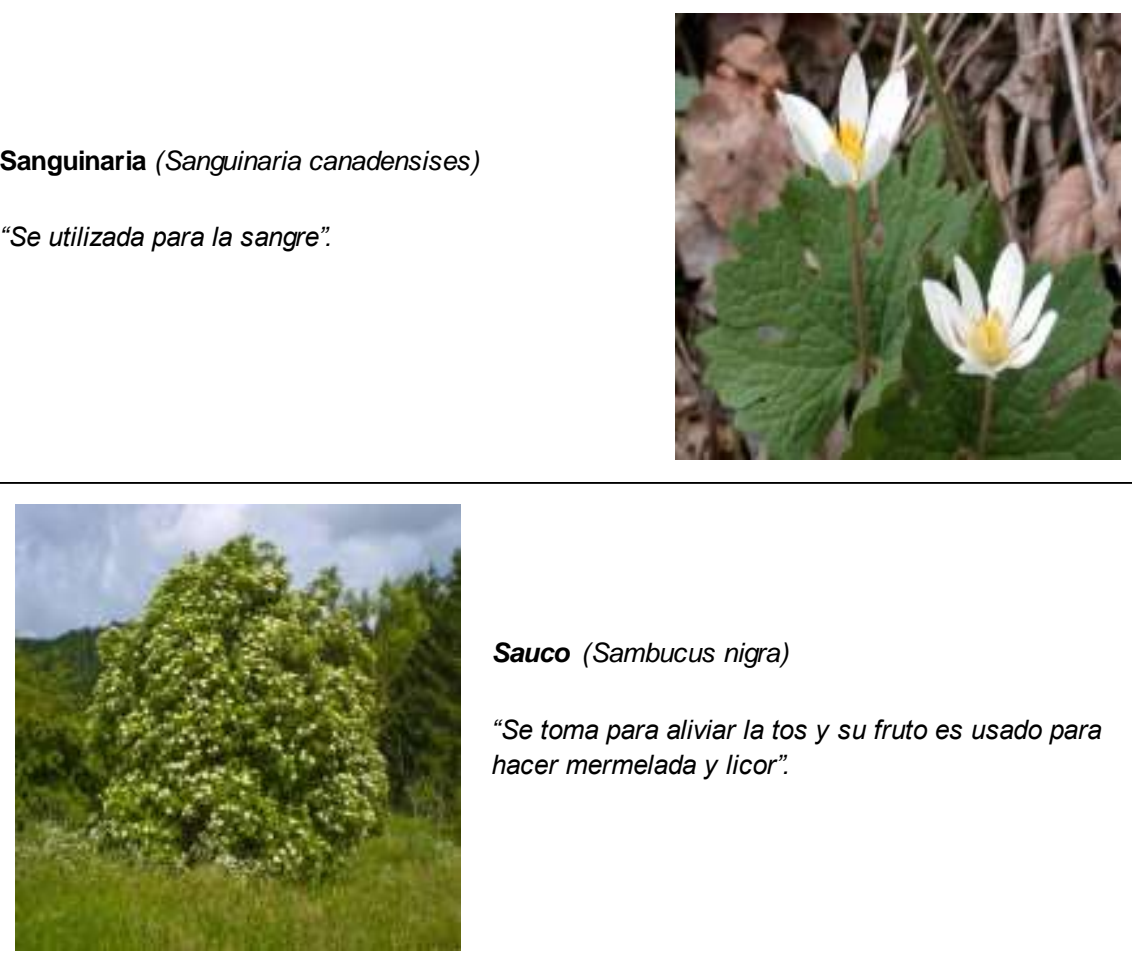

Sauco (Sambucus nigra)

"Se toma para aliviar la tos y su fruto es usado para hacer mermelada y licor".

Mutilla o chaura (Gaultheria mucronata)

"Fruto es dulce, la utilizan para hacer mermelada, tiene un sabor como el sauco".

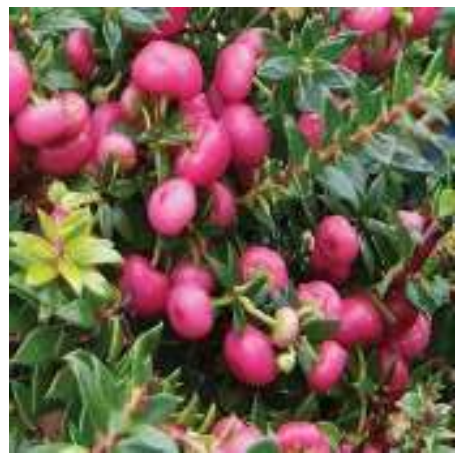




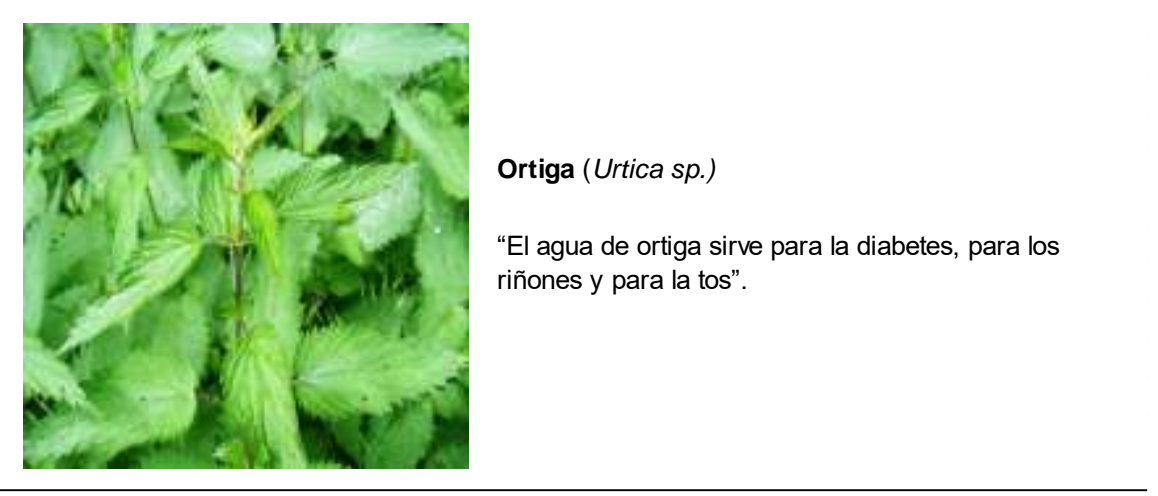

Calafate (Berberis microphylla)

"Tienen propiedades para la diabetes. Las productoras comentan que el calafate se está extinguiendo, por la falta de agua".

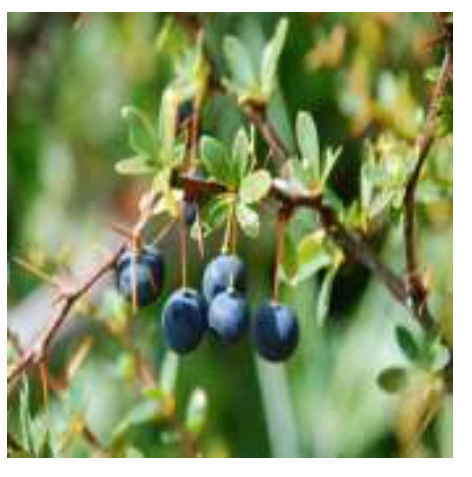

\section{CONCLUSIONES}

Los bosques de ñirre tienen importancia desde el punto de vista ambiental, social, ecológico y económico para las comunidades rurales de la región de Aysén, ya que de estos bosques se extrae una serie bienes y servicios que son relevantes para las personas y comunidades que habitan cercanos a ellos. Desde el punto de vista de la dinámica forestal, el ñirre es una especie rustica y resiliente, que se ha desarrollado y ha sobrevivido en escenarios de constantes disturbios naturales y antrópicos, que otras especies tal vez no podrían soportar.

Este trabajo permitió identificar alrededor de 70 especies vegetales y animales de uso no maderero presentes en los bosques de ñirre, con más de 100 diferentes usos para las personas, entre los que destacan usos medicinales, comestibles, tintóreos y ornamentales. Estas especies son colectadas por recolectores, principalmente mujeres, para consumo personal o comercialización.

Los PFNM en Chile han cobrado una gran importancia en el último tiempo, algunos son considerados como alimentos estrellas, como el calafate y el hongo comestible morchella. Ambos presentes en los bosques de ñirre en los meses de enero y octubre, respectivamente. Existen una proporción alta de especies de la familia Asteráceae que son utilizados para fines medicinales, entre ellas: la manzanilla, la melosa, la milenrama y el clavel del campo.

La especie principal de este tipo de bosques es el ñirre, especie con altos niveles de antioxidantes y que cuenta con múltiples usos no madereros, tales como partes vegetales de uso tintóreo, hojas y flores utilizadas para fabricación de licores, aceites esenciales y sumos para preparaciones gastronómicas.

La mujer dentro de la recolección de PFNM en los bosques de ñirre es protagonista. Más del $80 \%$ de personas que colectan estos productos son mujeres, que conocen el oficio y aplican técnicas de recolección sustentable de forma de no dañar el ecosistema que provee estos recursos. Por lo anterior, es necesario identificar o empadronar a estas recolectoras, con el fin de evitar la acción de personas que vienen de otras regiones, dejan los predios contaminados y colectan de forma no sustentable desconociendo la sabiduría tradicional de las recolectoras locales. 


\section{RECONOCIMIENTOS}

INFOR y los autores de esta publicación agradecen al Ministerio de Agricultura de Chile por el apoyo financiero que permiten la investigación de mediano y largo plazo en temas ligados a los PFNM. También agradecen la participación y la entrega de valiosa información por parte de las comunidades de recolectores que permitieron generar este documento.

\section{REFERENCIAS}

Armesto, J.J., Casassa, I. \& Dollenz, O. (1992). Age structure and dynamics of Patagonian Beech forests in Torres del Paine National Park, Chile. Vegetation, 98 Pp: 13-22. https://doi.org/10.1007/BF00031633.

Burns, S.L., Cellini, J.M., Lencinas, M.V., Martínez Pastur, G.J. \& Ruvera, S.M. (2010). Descripción de posibles híbridos naturales entre Nothofagus pumilio y Nothofagus antarctica en Patagonia Sur (Argentina). Bosque, 31(1): 9-16. https://doi.org/10.4067/S0717-92002010000100002.

CONAF. (2012. Actualización del Catastro y Evaluación de Recursos Vegetacionales Nativos. Región de Aysén.

Domínguez Díaz, E. (2012). Flora Nativa Torres del Paine. En: Domínguez Díaz, E. Flora Nativa Torres del Paine. Santiago: Ocho Libros Editores. Pp: 176-177.

Donoso, C. (1974). Manual de Identificación de Especies Leñosas del Bosque Húmedo de Chile, CONAF, Santiago, 168 p.

Donoso, C. (1987). Variación natural en especies de Nothofagus en Chile. Bosque, 8(2): 85-97. https://doi.org/10.4206/bosque.1987.v8n2-03.

Donoso, C. (2006). Las Especies arbóreas de los Bosques Templados de Chile y Argentina. Autoecología. Marisa Cúneo Ediciones, Valdivia, Chile. 678 p.

FAO. (2003). ¿Qué son los PFNM? Disponible en: http://www.fao.org/forestry/6388/es/. Consulta: 15 marzo, 2008.

Hansen, N., Codesal, P., Quinteros, P. \& Gallardo, C. (2013). Especies del sotobosque en ambientes de Nirre en Chubut. Ediciones INTA /EEA Esquel. Buenos Aires. 120 p.

Hoffmann, A. (1997). Flora silvestre de Chile, Zona Araucana: Árboles, arbustos y enredaderas leñosas. Ediciones Fundación Claudio Gay, Santiago, Chile. 258 p.

IREN-CORFO. (1979). Perspectivas de desarrollo de los recursos de la región de Aysén del General Carlos Ibáñez del Campo. Caracterización climática. $94 \mathrm{p}$.

Martínez, R. (2005). Elementos conceptuales que apoyan las decisiones sobre el fomento de productos forestales no maderables. 2 p.

Mc Queen, D.R. (1977). The ecology of Nothofagus and associated vegetation in South America. Tuatara, 22. Pp: 38-68.

Navarro Cerrillo, R.M., Rosenfeld, M., Pérez-Aranda, J., Padrón, E., Guzmán, J.R., Hernández Clemente, R. et al. (2008). Evaluación de la mortalidad de bosques de ñirre (Nothofagus antarctica) en la Patagonia chilena mediante imágenes Landsat TM y ETM+. Bosque, 29(1): 65-73. https://doi.org/10.4067/S0717-92002008000100008.

Ramírez, C., Correa, M., Figueroa, H. \& San Martin, J. (1985). Variación del hábito y hábitats de Nothofagus antarctica en el centrosur de Chile. Bosque, 6. Pp: 55-73. https://doi.org/10.4206/bosque.1985.v6n2-01.

Rodríguez, R., Mathei, O. \& Quezada, M. (1983). Flora Arbórea de Chile. Editorial Universitaria. Concepción, Chile. 408 p.

Romero, E.J. (1986). Fossil Evidence Regarding the Evolution of Nothofagus Blume. Annals of the Missouri Botanical Garden, 73. Pp: 276-283. https://doi.org/10.2307/2399113.

Salinas, J., Acuña, B., Uribe, A. \& Koch, L. (2015). Estudio de Regeneración Natural en Bosques de Nothofagus antarctica (Forster) Oerst. (Ñirre) Bajo Pastoreo Doméstico en la Región de Aysén. Instituto Forestal, Ministerio de Agricultura. Coyhaique, Chile. 50 p.

Salinas, J., Peri, P.L., Hepp, C. \& Acuña, B. (2017). Sistemas Silvopastorales en Bosques de Nirre (Nothofagus antarctica (G. Forst.) Oerst.) de la Región de Aysén. INFOR. Documento de Divulgación № 43.60 p.

Salinas, J., Moya, I. \& Gómez, C. (2018). Morchela (Morchella spp.) El Hongo de los Bosques Norpatagónicos de la Región de Aysén. Instituto Forestal, Chile. Documento de Divulgación N 45. 25 p. https://doi.org/10.52904/20.500.12220/28229.

Steinke, L., Premoli, A., Souto, C. \& Hedrén, M. (2008). Adaptive and neutral variation of the resprouter Nothofagus antarctica growing in distinct habitats in north-western Patagonia. Silva Fennica, 42. Pp: 177-188. https://doi.org/10.14214/sf.251. 